\title{
Cambios corneales producidos por la cirugía refractiva con excimer láser: revisión de tema
}

\author{
Virgilio Galvis* \\ Alejandro Tello* \\ Luis Carlos Jaramillo** \\ Ángela M. Castillo*** \\ Luis A. Pareja**** \\ Paul A. Camacho ${ }^{* * * * *}$
}

\begin{abstract}
*Médico Oftalmólogo. Centro Oftalmológico Virgilio Galvis. Docente. Universidad Autónoma de Bucaramanga. Facultad de Salud. Escuela de Medicina. Floridablanca. Colombia.

**Residente de Oftalmología IV Año. Universidad Autónoma de Bucaramanga. Facultad de Salud. Escuela de Medicina. Floridablanca. Colombia.

***Médico Oftalmólogo. Centro Oftalmológico Virgilio Galvis. Floridablanca. Colombia.

****Médico General. Centro Oftalmológico Virgilio Galvis. Floridablanca. Colombia.

***** Médico Epidemiólogo. Sub-Dirección de Investigación y Desarrollo. Fundación Oftalmológica de Santander FOSCAL. Docente. Universidad Autónoma de Bucaramanga. Facultad de Salud. Escuela de Medicina. Floridablanca. Colombia.

Correspondencia: Dr. Alejandro Tello. Dirección: Centro Oftalmológico Virgilio Galvis. Centro Médico Ardila Lulle. Torre A. Piso 3. Módulo 7. Teléfono: +57 7 6392929. FAX: 577 6392626. Floridablanca. Colombia. Correo electrónico: alejandrotello@gmail.com
\end{abstract}

\section{RESUMEN}

El auge de la cirugía refractiva con excimer láser en las últimas dos décadas se ha reflejado en que un grupo creciente de pacientes con antecedente de este procedimiento necesitan ahora cirugía de catarata. Debido a las modificaciones generadas en la córnea por la fotoablación con el láser, la determinación del poder corneal necesario para el cálculo del poder del lente intraocular a implantar en la cirugía de catarata, es difícil. Teniendo en cuanta estos aspectos se hace una revisión de las causas de estos errores de medición y los abordajes existentes para minimizar su impacto. MÉD.UIS. 2017;30(1):99-105.

Palabras clave: Láseres de Excímeros. Queratectomía Fotorrefractiva. Topografía de la Córnea. Córnea.

\section{Changes produced by corneal refractive surgery with excimer laser: a review}

\section{ABSTRACT}

The rise of excimer laser refractive surgery in the last two decades has been reflected in a growing group of patients with a history of this procedure who now need cataract surgery. Because the generated modifications to the cornea with the laser photoablation, determining the corneal power, necessary for calculating the power of the intraocular lens to be implanted during the cataract surgery, is difficult. We review the causes of these errors of measurement and existing approaches to minimize its impact. MÉD.UIS. 2017;30(1):99-105.

Keywords: Lasers, Excimer. Photorefractive keratectomy. Corneal topography. Cornea.

¿Cómo citar este artículo?: Galvis V, Tello A, Jaramillo LC, Castillo AM, Pareja LA, Camacho PA. Cambios corneales producidos por la cirugía refractiva con excimer láser: revisión de tema. MÉD. UIS. 2017;30(1):99-105.

\section{INTRODUCCIÓN}

La cirugía refractiva con excimer láser en sus diferentes modalidades, se emplea para corregir los defectos visuales (miopía, hipermetropía o astigmatismo) generando cambios en la curvatura de la córnea ${ }^{1,2}$. Los resultados actuales de estos procedimientos, con los equipos de última generación que poseen capacidades técnicas avanzadas, son buenos, y la satisfacción de los pacientes es muy alta3.5. Por otra parte, cuando estos pacientes llegan a la edad adulta avanzada, tienen el riesgo natural de presentar cambios seniles en el cristalino y de requerir cirugía de catarata ${ }^{6}$. El cálculo del poder del lente intraocular 
que se implanta luego de la extracción de la catarata, se fundamenta en una serie de fórmulas que tienen en cuenta siempre el poder corneal y la longitud axial. En estos casos se ha evidenciado que la medición del poder corneal realizado con los instrumentos usuales, no refleja el verdadero poder corneal del paciente operado de cirugía refractiva corneal con láser, y por ello se presentan errores en el cálculo del poder del lente intraocular cuando estos pacientes requieren cirugía de catarata. Se han diseñado múltiples métodos para tratar de compensar esos errores, con resultados bastante prometedores ${ }^{7,8}$. Sin embargo, la opción ideal sería poder obtener una medida precisa en el postoperatorio de la cirugía refractiva, con algún tipo de equipo o de método. En ese sentido han emergido nuevas tecnologías (tomógrafos corneales) que pueden aproximarse a obtener mediciones más reales de la córnea en el postoperatorio de cirugía con excimer láser.

Teniendo en cuenta el envejecimiento de la población, cada vez habrá un mayor número de pacientes que requieran cirugía de catarata y que tengan el antecedente de la cirugía corneal fotorrefractiva, por tanto, la disponibilidad de un método efectivo para solucionar este problema es necesario y pertinente. Investigadores como Holladay y coautores, Sónego-Krone y coautores, Gelender y Jaramillo y coautores han estudiado la medición del poder corneal en pacientes sometidos a cirugía fotorrefractiva utilizando parámetros obtenidos mediante diferentes tomógrafos corneales como el Pentacam (Queratometría Equivalente “EK”), el Orbscan II (Total-Optical Power "TOP" y Total-Mean Power "TMP") y el Sirius (Mean Pupil Power "MPP") y han encontrado que estas mediciones pueden servir de base para determinar de manera directa el poder corneal luego de cirugía fotorrefractiva ${ }^{9-12}$. El objetivo de este trabajo es realizar una revisión actualizada acerca de las causas de los errores de medición del poder corneal luego de cirugía fotorrefractiva y de los métodos existentes para determinar de manera directa el poder corneal real luego de estos procedimientos.

\section{Metodología}

La obtención de la información se hizo mediante consulta de la bases de datos como Medline/PubMed para artículos que se publicaron en cualquier idioma hasta el día 12 de marzo de 2016. Los términos de búsqueda que se usaron (en idioma inglés) fueron: "poder corneal”, “queratometría”, “topografía corneal”, “tomografía corneal”, "poder del lente intraocular" empleando la herramienta de búsqueda avanzada, con la limitante de que los términos estuvieran incluidos dentro del título o el resumen de los artículos y adicionalmente que también incluyeran en el título o el resumen los términos "Excimer láser" o "cirugía refractiva”. Adicionalmente, se realizó una búsqueda en cada página de las revistas nacionales clasificadas en Publindex como categorías $A 1, A 2$ y $B$ con los términos "refractiva" o "excimer".

En total se identificaron 899 publicaciones. Se revisaron los títulos para seleccionar los que se relacionaran directamente con la determinación de los cambios corneales generados por la cirugía refractiva y su impacto en el cálculo del poder del lente intraocular. Luego de la revisión de los resúmenes se seleccionaron 29 artículos para ser revisados en forma completa. Finalmente, se escogieron para ser incluidos dentro de la revisión de tema 17 artículos de este grupo. Se adicionaron cuatro artículos más, procedentes de las citaciones de estos y otros dos trabajos aún no publicados por los autores del actual artículo (uno enviado y uno aceptado), los cuales se consideraron pertinentes.

\section{Marco Teórico}

\section{CIRUGÍA REFRACTIVA CON EXCIMER LÁSER: PRINCIPIOS Y TÉCNICA}

El principio básico de la cirugía refractiva con excimer láser es la de cambiar la forma de la cara anterior de la córnea retirando una cantidad muy precisa de tejido estromal. Esto se logra aplicando una energía lumínica muy intensa, que causa una ruptura de los enlaces de las moléculas del tejido y por lo tanto los descompone, originando lo que se conoce como una fotoablación. Cuando el ojo sufre de miopía, es decir la imagen de un objeto lejano se está formando antes de la retina, la cirugía consiste en retirar tejido del área central, de manera que la córnea en esa zona se vuelva menos curva. Al disminuir así el poder de convergencia de los rayos de luz, el foco se desplazará hacia atrás y llegará a la mácula. Por el contrario, en un ojo hipermétrope la fotoablación con el excimer láser se realiza en la media periferia de la córnea, de manera que el centro de la córnea se torna mas curvo. Como en la hipermetropía la imagen se forma detrás de la retina, al ser la córnea más curva, y por ende más poderosa, el foco se desplazará hacia delante, llegando a la retina (Ver Figura 1). 


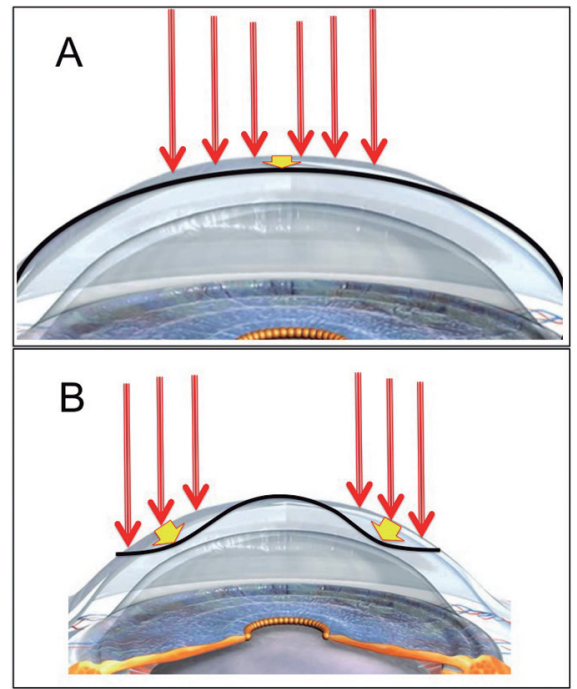

Figura 1. A: En el caso de miopía el láser (flechas rojas) se aplica en el centro de la córnea, de manera que elimine una cantidad de tejido en esa zona (flecha amarilla), lo que hace que el perfil de la córnea cambie y llegue a ser menos curva (línea negra). B: En el caso de hipermetropía el láser (flechas rojas) se aplica en la media periferia de la córnea, de manera que elimine una cantidad de tejido en esa zona (flechas amarillas), lo que hace que el perfil de la córnea cambie y llegue a ser más curva en el centro (línea negra).

Fuente: autores

Existen dos técnicas básicas de cirugía refractiva con excimer láser. La primera consiste en realizar inicialmente un corte que genera un colgajo de tejido corneal de aproximadamente 130 micras de espesor. Al levantar este colgajo que incluye el epitelio, la membrana de Bowman y el estroma superficial, se expone el estroma corneal medio, y es allí en donde se aplica la energía del láser. Esta técnica se denomina queratomileusis asistida con excimer láser, y se conoce como LASIK por sus siglas en inglés. La otra alternativa es la aplicación directa del láser sobre la superficie de la córnea, en lo que se conoce como técnicas de ablación de superficie. Dentro de estas técnicas se encuentra la queratotomía fotorrefractiva (conocida como PRK por sus siglas en inglés) y la queratotomía fotorrefractiva transepitelial (conocida como TRANS-PRK por sus siglas en inglés). La diferencia fundamental con el LASIK es que estas técnicas no se acompañan de ningún corte y el láser se aplica sobre la membrana de Bowman después de haber retirado mecánicamente el epitelio (en el caso de la PRK) o directamente sobre el epitelio corneal (en el caso de la TRANS-PRK). Los resultados de las dos técnicas son equivalentes en los defectos bajos o moderados. En los defectos mas altos, la ablación de superficie tiene un poco mas de riesgo de generar cicatrización corneal, y se prefiere el LASIK, si es que el espesor de la córnea lo permite.

\section{CÁLCULO DEL PODER DE LOS LENTES INTRAOCULARES}

Hasta mediados del siglo XX a los pacientes que se operaban de catarata se les extraía el cristalino opaco y no se les implantaba un lente artificial (lente intraocular) que compensara el poder de convergencia óptico del cristalino. Los pacientes quedaban áfacos (sin cristalino ni lente intraocular) y requerían el uso de unas gafas con un poder de aumento positivo muy alto. A finales de la década de los años cuarenta un oftalmólogo del Reino Unido que trabajaba con la Real Fuerza Aérea (el Dr. Harold Ridley) desarrolló e implantó el primer lente intraocular para reemplazar al cristalino ${ }^{13-15}$. El proceso para que este tipo de prótesis se desarrollara hasta lograr un buen desempeño clínico, seguro y efectivo, tomó alrededor de tres décadas. Actualmente, los avances en las técnicas quirúrgicas para extraer el cristalino (microcirugía con facoemulsificación) y la calidad óptica de los nuevos lentes intraoculares, han permitido que la cirugía de catarata se convierta en una cirugía muy segura y con resultados refractivos predecibles en la mayoría de los pacientes ${ }^{16}$.

Sin embargo, para alcanzar esto es necesario realizar mediciones preoperatorias precisas para calcular el poder del lente intraocular usando las fórmulas biométricas. Actualmente, se emplean frecuentemente las fórmulas de tercera (SRK/T, Hoffer Q y Holladay 1) y de cuarta generación (Haigis, Holladay 2, Olsen, Barrett II) para calcular el poder del lente intraocular y los resultados son muy satisfactorios en ojos vírgenes ${ }^{16}$.

Para determinar el poder del lente intraocular a implantar, de manera que el ojo operado quede lo mas cerca posible de la emetropía, se requiere conocer al menos dos datos: la longitud axial del ojo y el poder de la córnea. Basándose en estos datos (y otros adicionales en algunos casos) las fórmulas intentan predecir la posición en que se ubicará el lente intraocular, y posteriormente calcular su poder dióptrico ideal en un segundo paso $7,8,17$.

En los últimos años la investigación y el interés en el cálculo del poder del lente intraocular en pacientes que se han sometido a cirugía refractiva con excimer láser se ha incrementado debido al gran número de estos procedimientos refractivos que se han realizado en las últimas dos décadas y a que muchos de esos pacientes están empezando a desarrollar cataratas 
seniles y muchos otros la desarrollarán en el futuro, requiriendo implante de lentes intraoculares, cuyo poder es difícil de calcular en estos casos por razones que se analizaran más adelante. Adicionalmente, estas son personas que en general alcanzaron muy buena agudeza visual después de su procedimiento refractivo y por lo tanto tienen expectativas muy altas con respecto al resultado final de la cirugía de catarata.

Son diversos los factores que afectan la precisión de los cálculos biométricos en esta situación, y por ello se requiere de una serie de consideraciones especiales con el fin de evitar sorpresas refractivas ${ }^{18,19}$.

\section{DETERMINACIÓN DEL PODER DE LA CÓRNEA EN UN OJO NORMAL}

El poder de la córnea se determina en la clínica con el empleo de instrumentos de reflexión y de enfoque manual (el queratómetro) o equipos automatizados de reflexión (topógrafos), o que usan algún tipo de hendidura óptica (tomógrafos). Todos los queratómetros, topógrafos y tomógrafos miden el radio de curvatura de la cara anterior de la córnea en milímetros y convierten este dato a poder en dioptrías.

La relación entre el poder óptico y el radio de una superficie esférica se expresa en la siguiente fórmula de acuerdo a la óptica Gaussiana con la aproximación paraxial:

$$
\mathrm{P}=\mathrm{n}-1 / \mathrm{r}
$$

Donde $\mathrm{P}=$ poder óptico en dioptrías, $\mathrm{n}=$ índice de refracción del lente y $r=$ radio en metros.

Los queratómetros, topógrafos y tomógrafos tradicionalmente se usan para determinar el poder total de la córnea, basándose únicamente en la medida del radio de curvatura de la cara anterior, usando en la fórmula un valor acuñado en lugar del índice de refracción, de manera que compense el poder de la cara corneal posterior (cuyo radio de curvatura era muy difícil de medir cuando se empezó a determinar el poder de la córnea a finales del siglo XIX, y aún es un reto para los equipos). Este valor $(n=1.3375)$ es conocido como el "índice queratométrico"s. El poder promedio de la córnea medido con este abordaje es de aproximadamente 43,00 D.

Si se quiere determinar el poder total real de la córnea (no el aproximado usando el índice queratométrico), se debe calcular el poder de las dos superficies refractivas (anterior y posterior) y tomar en consideración el espesor del lente, que en este caso corresponde a la paquimetría promedio, aplicando la fórmula de la lente gruesa.

$$
\mathrm{P}=(\mathrm{n} 2-\mathrm{n} 1) / \mathrm{r} 1+(\mathrm{n} 3-\mathrm{n} 2) / \mathrm{r} 2-\mathrm{T}
$$

Donde $\mathrm{P}=$ poder de la córnea (Dioptrías); $\mathrm{n} 1=$ índice de refracción del aire $(1,00) ; \mathrm{n} 2$ = índice de refracción de la córnea $(1,376)$; n3=índice de refracción del humor acuoso $(1,336) ; \mathrm{r} 1$ = radio de la cara anterior de la córnea (metros); r2=radio de la superficie posterior de la córnea (metros); $\mathrm{T}=$ efecto refractivo de la paquimetría en la fórmula del lente grueso ${ }^{7,18-20}$.

Teniendo en cuenta esta fórmula sería posible calcular el poder de la córnea, si los radios de curvatura de la cara anterior y posterior se conocieran. Esto es factible con los tomógrafos corneales modernos, que pueden medir el radio de curvatura tanto de la superficie anterior como de la superficie posterior de la córnea ${ }^{19-22}$.

\section{CAUSAS DE ERRORES BIOMÉTRICOS EN OJOS POST-CIRUGÍA FOTORREFRACTIVA}

Teniendo en cuenta las consideraciones anteriores de cómo se establece el poder total de la córnea en un ojo normal, se pueden entender entonces las variaciones que se presentarán en un ojo con cirugía queratorrefractiva previa. El primer factor de error en la determinación del poder corneal con la queratometría manual o automatizada es que los instrumentos realizan la medición de la superficie anterior de la córnea en una zona de aproximadamente $2,6 \mathrm{~mm}$ (autoqueratómetros, topógrafos y tomógrafos) a 3,omm de diámetro (queratómetro manual) ya que no es posible hacer una medida del área directamente sobre el ápice. En una córnea normal la diferencia de esta zona un poco más periférica con el centro es tan pequeña que no genera un error significativo, ya que a esa distancia existe un perfil corneal casi esférico o mínimamente prolato (es decir algo más curvo en el centro). Sin embargo, en una córnea operada con excimer láser se ha alterado ese perfil, y el centro será menos curvo que el punto medido (tendencia a la oblaticidad) en un ojo operado de miopía y significativamente más curvo (mayor prolaticidad) en un ojo operado de hipermetropía. La magnitud del error será mayor en ojos operados de miopía, porque al tener una curva más plana, la medida del queratómetro se realizará en una zona aún más alejada del ápice corneal ${ }^{8}$ (Ver Figura 2). 
ERERO - ABRIL

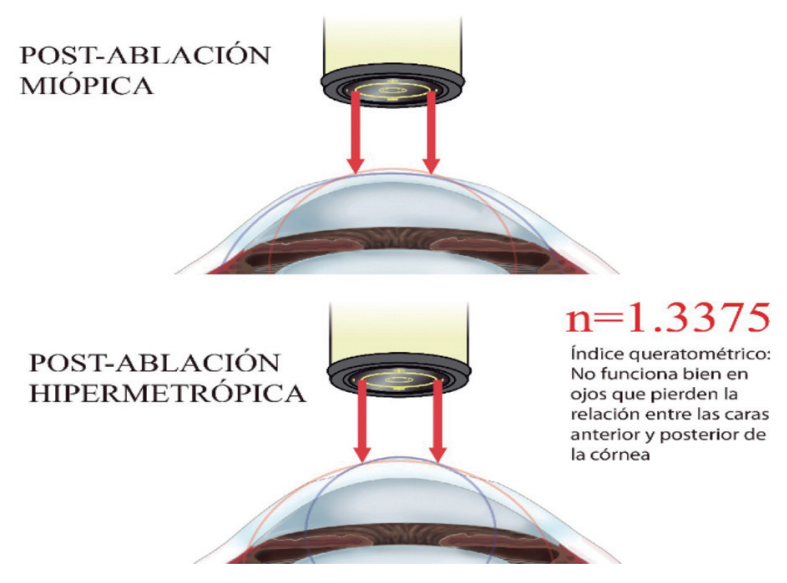

Figura 2. Al realizar la medida en la zona paracentral, los queratómetros y topógrafos corneales se equivocan luego de cirugía refractiva con láser. La curvatura corneal en una córnea no operada tiende a ser casi esférica en el centro, no habiendo diferencia significativa con la curvatura paracentral. Sin embargo, en las córneas operadas si existen diferencias generadas por la ablación de tejido.

Fuente: autores.

Además, como ya se indicó, para calcular el poder corneal total conociendo únicamente el radio de curvatura de la cara anterior, los queratómetros y topógrafos/tomógrafos emplean el llamado índice queratómetrico $(1,3375)$ el cual funciona suficientemente bien en córneas normales. Con la cirugía fotorrefractiva, sin embargo, el escenario se modifica sustancialmente ya que el procedimiento genera el cambio en el poder de la córnea, modificando su curvatura en la cara anterior, pero sin causar un cambio significativo en la cara posterior. Al perderse la relación entre la cara anterior y la cara posterior de la córnea, el índice queratométrico ya no es válido en estos pacientes, y este factor de imprecisión es aditivo al ya mencionado de la medición paracentral, para causar en últimas un error significativo en la determinación del poder corneal en los ojos operados de cirugía refractiva.

Teniendo en cuenta este error en la determinación del poder corneal y otro error adicional que se presenta con las fórmulas biométricas de tercera generación en la estimación de la posición efectiva del lente, si se emplea la queratometría estándar y una fórmula de tercera generación, sin realizar ajustes, en los pacientes operados de miopía vamos a tener una sobreestimación del poder de la córnea central y una subestimación de la distancia a la que se implantará el lente (posición efectiva del lente). Estos dos errores son aditivos, llevando finalmente a una subestimación de poder del lente y a un resultado postoperatorio hipermetrópico luego de la cirugía de catarata. En los pacientes operados de hipermetropía vamos a tener una subestimación del poder de la córnea central y una sobrestimación de la distancia a la que se implantará el lente (posición efectiva del lente), errores que son aditivos llevando finalmente a una sobrestimación de poder del lente y a un resultado postoperatorio miópico luego de la cirugía de catarata ${ }^{8}$. Por todo esto el cálculo del poder del lente intraocular que va a ser implantado en pacientes con cirugía refractiva se ha convertido en un reto para los especialistas.

\section{MÉTODOS DE DETERMINACIÓN DEL PODER CORNEAL LUEGO DE CIRUGÍA FOTORREFRACTIVA CON EXCIMER LÁSER}

Con el fin de solucionar los errores ya mencionados, se han propuesto múltiples métodos. Dentro de estos métodos se encuentra el denominado de "Historia Clínica" el cual fue descrito por Holladay inicialmente para ser aplicado en pacientes operados con cirugía refractiva con bisturí de diamante (queratotomía radial), como se realizaba en las décadas de 1970 y 1980. Esta alternativa de cálculo aún se considera la "regla de oro" en casos de pacientes operados de cirugía refractiva ${ }^{23,24}$. Este método se basa en un principio sencillo que consiste en calcular el cambio de refracción generado por la cirugía, ajustar ese cambio al nivel del plano corneal, y restarle o sumarle, según sea el caso, ese cambio al poder corneal medido en el preoperatorio. La debilidad de este método es que requiere información completa tanto del estado pre como del post-operatorio. Se han diseñado otros métodos, y actualmente se han incluido en calculadores online, con diversos abordajes para compensar el error de esta medición. Algunos de estos métodos no requieren información previa y se basan en las medidas de la córnea postoperatoria realizadas con tomógrafos y ajustada con algunos factores de corrección ${ }^{25}$.

Jaramillo y coautores recientemente realizaron un estudio de determinación del poder corneal en pacientes con antecedente de cirugía refractiva con láser basándose en las mediciones postoperatorias realizadas con los tomógrafos corneales Orbscan Ilz y Sirius ${ }^{12}$. En este estudio se pudo concluir que el poder corneal obtenido mediante el mapa Mean Pupil Power del tomógrafo Sirius, luego de la aplicación de una sencilla fórmula de regresión, puede ser muy similar al valor obtenido a partir de la queratometría derivada por el método de la "Historia Clínica", en pacientes que han sido sometidos a cirugía fotorrefractiva con excimer láser. La diferencia fue 
tan baja entre el poder corneal calculado por el mapa Mean Pupil Power y el derivado del método de la "Historia Clínica", que clínicamente no es significativa e inclusive existe la posibilidad de su utilización de forma directa como valor de poder corneal real postoperatorio en pacientes con cirugía fotorrefractiva con excimer láser.

Con el tomógrafo Orsbscan Ilz luego de valorar mapas que incluían diferentes zonas corneales (desde 1 hasta $4 \mathrm{~mm}$ de diámetro) se encontró que para el "Mapa de Poder Promedio Total" generado por ese equipo, la zona de $3 \mathrm{~mm}$ tuvo la mejor correlación con el método de la historia clínica. Para el "Mapa de Poder Óptico Total" con este equipo la zona de 3,5 mm de diámetro tuvo la mejor correlación con el método de la historia clínica, siendo esta mejor que la alcanzada por el "Mapa de Poder Promedio Total" 12.

En el estudio de Holladay y coautores publicado en 2009, donde se utilizó el valor EK del tomógrafo Pentacam para medir el poder corneal central se obtuvo una desviación media de -0,06 \pm 0,56 D en 100 pacientes sometidos a LASIK $^{10}$. Por otro lado, Jaramillo y coautores utilizando el Mean Pupil Power del tomógrafo Sirius observaron una desviación media con respecto al método de la Historia Clínica”, en los ojos miopes, de 0,22 D. Analizando el subgrupo de miopes mayores de 5 D la desviación media fue mucho menor de 0,04 D (sin diferencia estadísticamente significativa), pero en los miopes menores de $5 \mathrm{D}$ la desviación media fue mayor 0,28 $D$, alcanzado significancia estadística $(p=0.001)$. Las diferencias en ojos hipermétropes (-0,15 D) y con astigmatismo mixto (-0,03 D) no alcanzaron significancia estadística ${ }^{12}$.

Por otra parte, Sónego-Krone y coautores en un trabajo publicado en 2004, utilizaron múltiples mapas de poder corneal obtenidos por el Orbscan II, encontrando que la utilización de los mapas llamados Total-Mean Power y el Total-Optical Power evalúan de forma precisa el poder corneal en corneas luego de LASIK miópico. Adicionalmente, se encontró que el Total-Mean Power es útil en el área de $2 \mathrm{~mm}$ de diámetro central con una desviación media de 0,38 D, mientras que el Total-Optical Power realiza una mejor evaluación del poder corneal en 3 y 4 mm de diámetro central con una desviación media de 0,26 D y-0,08 D, respectivamente ${ }^{9}$.

En un estudio por Gelender publicado en el 2006, en ojos sometidos a LASIK miópico se derivó el poder corneal a partir de los mapas del equipo Orbscan II denominados "Mean Power" y se encontró que las áreas de 1,5 y $2 \mathrm{~mm}$ centrales fueron las más precisas para realizar esta medida ${ }^{11}$.

\section{CONCLUSIÓN}

Los nuevos equipos que emplean una luz de hendidura para determinar el radio de curvatura tanto de la superficie córneal anterior como de la posterior (como el Orbscan IIz y el Sirius), son una buena opción para obtener información directa sobre el poder corneal real en ojos operados de cirugía fotorrefractiva. Esto podrá disminuir una fuente de error en los cálculos del poder del lente intraocular utilizando fórmulas biométricas cuando estos pacientes desarrollen catarata senil y deban ser sometidos a cirugía de facoemulsificación mas implantación de lente intraocular.

\section{REFERENCIAS BibLIOGRÁFICAS}

1. Galvis V, Tello A, Aparicio JP. Excimer laser Refractive Surgery: A Review. Med UNAB. 2007;10:99-105.

2. Galvis V, Tello A. Defectos Refractivos y su Corrección Quirúrgica: Revisión. MED UIS. 2009;22(2):158-73.

3. Bahrawy M El, Alió JL. Excimer laser 6 th generation : state of the art and refractive surgical outcomes. Eye Vis. 2015;2(6):1-9.

4. Tanzer DJ, Brunstetter T, Zeber R, Hofmeister E, Kaupp S, Kelly N, et al. Laser in situ keratomileusis in United States Naval aviators. J Cart Refract Surg. 2013;39(7):1047-58.

5. Brown M, Schallhorn S, Hettinger K, Malady S. Satisfaction of 13,655 patients with laser vision correction at 1 month after surgery. J Refract Surg. 2009;25(7):S642-6.

6. Galvis V, Tello A, Carreño N. El cristalino para el médico general. Med UNAB. 2008;11:225-30.

7. Hodge C, Mcalinden C, Lawless M, Chan C, Sutton G, Martin A. Intraocular lens power calculation following laser refractive surgery. Eye Vis. 2015;2(7):1-8.

8. Haigis W. Intraocular Lens Calculation After Refractive Surgery. Eur Ophthalmic Rev. 2012;6(1):21-4.

9. Sónego-Krone S, López-Moreno G, Beaujon-Balbi O V., Arce CG, Schor P, Campos M. A Direct Method to Measure the Power of the Central Cornea After Myopic Laser In Situ Keratomileusis. Arch Ophthalmol. 2004;122:159-66.

10. Holladay J, Hill W, Steinmueller A. Corneal Power Measurements Using Scheimpflug Imaging in Eyes With Prior Corneal Refractive Surgery. J Refract Surg. 2009;25(10):862.

11. Gelender, H. Orbscan II - assisted intraocular lens power calculation for cataract surgery following myopic laser in situ keratomileusis. Trans Am Ophthalmol Soc. 2006;104:402-13.

12. Jaramillo LC, Galvis V, Tello A, Camacho P. Poder corneal luego de cirugía refractiva: determinación con un tomógrafo corneal. Med UNAB. 2016;Enviado.

13. Apple DJ, Sims J. Harold Ridley and the Invention of the Intraocular Lens. Surv Ophthalmol. 1996;40(4):279-92.

14. Teichmann K. Landmarks in the evolution of cataract surgery. Surv Ophthalmol. 2000;44(6):541.

15. Ashwin P, Shah S, Wolffsohn J. Advances in cataract surgery. Clin Exp Optom. 2009;92(4):333-42.

16. Gale RP, Saldana M, Johnston RL. Benchmark standards for refractive outcomes after NHS cataract surgery. Eye. 2009;92(4):149-52.

17. Chen X, Yuan F, Wu L. Metaanalysis of intraocular lens power calculation after laser refractive surgery in myopic eyes. J Cart 
Refract Surg [Internet]. ASCRS and ESCRS; 2016;42(1):163-70. Available from: http://dx.doi.org/10.1016/j.jcrs.2015.12.005

18. Savini G, Barboni P, Carbonelli M, Ducoli P, Hoffer KJ. Intraocular lens power calculation after myopic excimer laser surgery: Selecting the best method using available clinical data. J Cart Refract Surg [Internet]. ASCRS and ESCRS; 2015;41(9):1880-8. Available from: http://dx.doi.org/10.1016/j.jcrs.2015.10.026

19. Kim D, Kim M, Wee W. Estimation of intraocular lens power calculation after myopic corneal refractive surgery: using corneal height in anterior segment optical coherence tomography. Korean J Ophthalmol. 2015;29(3):195-202.

20. Canovas C, van der Mooren M, Rosén R, Piers P, Wang L, Koch D. Effect of the equivalent refractive index on intraocular lens power prediction with ray tracing after myopic laser in situ keratomileusis. J Cart Refract Surg. 2015;41(5):1030-7.

21. Olsen T. Calculation of intraocular lens power : a review. Acta
Ophthalmol Scand. 2007;85(5):472-85.

22. Savini G, Carbonelli M, Sbreglia A, Barboni P, Deluigi G Hoffer KJ. Comparison of anterior segment measurements by 3 Scheimpflug tomographers and 1 Placido corneal topographer. J Cataract Refract Surg. 2011;37:1679-85.

23. Holladay, JT. Surgery, Consultations in Refractive Surgery: IOL calculations following radial keratotomy. Refract Corneal Surg. 1989;5:203.

24. Holladay J, Galvis V, Tello A. Re : Wang et al .: Comparison of newer intraocular lens power calculation methods for eyes after corneal refractive surgery. Ophthalmology. 2016;123(9):e55-6.

25. Wang L, Tang M, Huang D, Weikert MP, Koch DD. Comparison of Newer Intraocular Lens Power Calculation Methods for Eyes after Corneal Refractive Surgery. Ophthalmology [Internet]. Elsevier; 2015;122(12):2443-9. Available from: http://dx.doi.org/10.1016/j. ophtha.2015.08.037 